\title{
A SYSTEMATIC REVIEW OF STUDIES RELATING TO THE PSYCHOLOGICAL WELL-BEING OF DOCTORS AND ALLIED HEALTHCARE PROFESSIONALS DURING THE COVID-19 PANDEMIC
}

\author{
Manna Dey \\ North South University, Bangladesh \\ E-mail: deymanna113@gmail.com \\ Dennis Relojo-Howell \\ Psychreg, United Kingdom \\ E-mail: dennis@psychreg.org
}

\begin{abstract}
Doctors and other allied healthcare professionals are constantly exposed to stressful situations that can undoubtedly negatively affect their psychological well-being. This precarious situation has been further exacerbated due to the COVID-19 pandemic. This study aims to explore the mental stress and trauma among doctors, in light of the pandemic. A number of relevant studies (quantitative, qualitative, mixed methods, and case studies) have been gathered to present a global trajectory of the responses of the doctors in the pandemic, and, as a consequence how this has impacted their psychological well-being. Collated findings suggest a need for a more nuanced and global approach, which is necessary towards addressing the immediate and long-lasting effects of the pandemic within the medical community. This study also provides a theoretical framework that can be used to design psychological interventions which can target the healthcare profession.
\end{abstract}

Keywords: community psychology; COVID-19, healthcare professionals, psychological well-being; systematic review

\section{Introduction}

In the present systematic review, a literature search was carried out with the goal of reviewing literature to elucidate the psychological well-being of doctors and other allied healthcare professionals during the COVID-19 pandemic. A wide search was made using the internet, the books of the National Institute of Preventive and Social Medicine, and local libraries. The researchers received aid from several sources. This review was conducted in leading and representative papers from available sources. In several countries, the psychological well-being of physicians has been previously investigated. A cross-sectional study (Chekole et al., 2020) was carried out from 15th March to 15th April 2020 in Egypt on the demographic and mental health of anaesthesiologists and ICH doctors who work in university hospitals in Cairo. The stress level was evaluated by the stress level perceived (PSS-10). The survey was completed by 193 out of 315 contacted doctors. Most were males and between 26 and 45 years of age. The PSS-10 median scoring of 21 in the record group and 18 in the consultant group 
were statistically significant for a substantial number of the participants, whereby $65 \%$ experienced extreme psychological distress during this acute circumstance $(p=03) ; 70 \%$ of healthcare workers Vol. 15, No. 1, 2021 have experienced psychological problems in a comparable study in Wuhan, China, released recently during this pandemic.

As evidenced by related studies, physicians have a complex psychological reaction to an epidemic of contagious viruses. The causes of anguish consist of thoughts of exposure or inevitability, and concerns about the spread of the disease among relatives and colleagues. The point is that COVID-19 is a disease transmissible to humankind, concurrent with unbelievable morbidity (Galea \& Keyes, 2020). Awareness of self-harm may increase fatality. Likewise, expected supply shortages, personal protective gear, and no clear methods to address dubious and proven COVID-19 cases add to clinicians' stress and concern (Khandelwal, 2020). The results of this study confirmed the concepts, since the majority of participants were concerned that they could transmit the disease to their loved ones, and a lack of an unambiguous protocol on how to deal with suspected or confirmed COVID-19 cases annoyed them, causing huge stress and insufficient availability of the personal protective equipment (PPE).

\section{Related Study to Psychological Evaluation of Doctors}

A further study intended at analysing the immediate psychological effect on clinicians working in Dhaka Medical College Hospital, the largest COVID-19 facility in the country. As the COVID-19 outbreak is affecting healthcare workers worldwide in an unforgettable way, it comes as no surprise that Bangladeshi healthcare workers' mental well-being is also an inseparable risk. The cross-sectional study (Pilao et al., 2017; Sanghera et al., 2020) was carried out by participating physicians from 31st May to 3rd June 2020 at a single clinic. The Athens Insomnia Scale and Hospital Anxiety Depression Score investigated the mental health characteristics (HADS). A total of 192 questionnaires collected were obtained (response rate was $64.43 \%$ ), with depression and anxiety symptoms in about $50 \%$ of participating doctors, respectively. Of those who answered to sleeplessness, 104 (54.17\%) responded. Roughly $56 \%$ of the respondents saw the COVID-19 as the most glaring threat to COVID-19 as highly contaminating.

Doctors have significant levels of work stress even under normal circumstances, but many do not like to reveal problems with mental health or seek assistance for stigma for a reason that is widely mentioned (Cohen \& Patten, 2005). The COVID-19 situation puts more strain on physicians and the overall health system, and research shows that such strain is more susceptible to psychological discomfort. Doctors in Bangladesh faced rising obstacles which they did not face during the COVID-19 outbreak (Chowdhury et al., 2021). Decisions must be made quickly, ranging from efficient trials and isolation of infected patients to choosing if the departments and operating theatres can be shut down when a patient or staff is tested positive. There has been an enormous push for prompt and successful diagnosis (Relojo-Howell, 2020), isolation, and treatment, especially with intensive screening in the public and media. This corresponds to experience in other countries (Chavez-Valdez et al., 2019). Studies have consistently shown that the occupation of healthcare is more stressful than the general population at work, even under normal conditions, and stress is related both with physical and mental health problems. Studies have also demonstrated that it is difficult for many doctors to tell their employers about mental health problems (Antoniou et al., 2003; Coffey, 1999; Laposa et al., 2003). The most frequently mentioned reasons are stigma and feared damage to future opportunities of working.

In the event of a pandemic, a considerable number of patients requiring treatment are increasing, and the health resources and staff are placed under pressure. In addition, healthcare professionals perceive a higher risk of exposure to the most deficient patients (Vetter et al., 2008) - adding additional stress. The lack of personal protective gear (PPE) that could occur during a pandemic compound is stress. The greater possibility that families of health professionals would be infected at the frontline is an additional stressor. Pandemic data from 2009 suggest that among at least one of their family members $20 \%$ of physicians and nurses with symptoms have reported symptoms (Hui et al., 2010). This poll shows a limited depression of $35.42 \%$ and depression of $16.66 \%$. A survey in Wuhan, China found that the symptoms of stress, sadness and anxiety were $29.8 \%, 13.5 \%$, and $24.1 \%$ respectively (Fu et al., 2020). Depression and anxiety of the health care workforce was relatively low and could connect to the various assessments employed by the Tongi Hospital managers 
OF PSYCHOLOGY

IN THE $21^{\text {st }}$ CENTURY Vol. 15, No. 1, 2021

to COVID 19 in their early stages, and psychological protection measures undertaken (Acharya \& Relojo, 2017). Researchers found $22.92 \%$ of doctors serving at dedicated COVID 19 hospitals are anxious whereas roughly $30 \%$ had limited anxiety. Our study also revealed that a large proportion of doctors suffer from sleep problems (31.2\%) and sleep problems (54.17\%) (Spoorthy et al., 2020). As a result, psychological pressure and psychiatric morbidity rates are high among health workers who deal with COVID-19, similar to the scenario during epidemics of SARS and H1N1. These affects ordinary clinical practices and the sensation of control loss and the associated concern that health care services would be destabilised has caused anxiety (Pinto-Coelho \& Relojo, 2017; Pilao et al., 2019; Relojo-Howell \& Stoyanova, 2019) and despair among healthcare professionals, which is a feature of epidemics not uncommon.

\section{Healthcare Workers' Study on Acute Stress}

This cross-sectional study examines the volume and direction of the response ability of the healthcare professionals who have been suffering from anguish due to care for patients with COVID-19 to meet the future waves of COVID-19 (Marques et al., 2021). The survey was completed by a nonrandomized sample of 685 professionals (doctors, nurses, and health workers). The acute stress of health professionals Caring COVID-19 scale measures both the frequency and intensity of stress responses (EASE). The stress reactions vary by the daily death rate per territory and by the evolutionary stage of the COVID-19 outbreak determined by the Kruskal-Wallis and the Mann-Whitney-U tests. The EASE score averaged 11.1 out of 30 (SD 6.7). 44.2\% of the subjects showed good emotional adaptation, $27.4 \%$ were bearable, $23.9 \%$ were medium-high and $4.5 \%$ were intense acute stress. In the most impacted areas (12.1 vs 9.3, $p=0.003)$ and in the disillusionment phase $(12.7 \mathrm{vs} 8.5$, heroic 10.2 and 9.8 honeymoon, $p=0.001$ ) there were more stress reactions.

Acute stress was most reflected by the inability to separate from work and the concern that loved ones would get infected (Brooks et al., 2020). The declarations that most likely discriminate against professionals whose condition prevents them from continue their care are losing empathy for patients' suffering and a dread of illness. This study confirms what in other studies has been stated that about $5 \%$ of the professionals suffering from significant acute stress are due to care for COVID-19 patients. Taking these data into account, the objectives of the COVID- 19 pandemic interventions must take into consideration that around a quarter of professionals would need help to not develop to extremely accurate scenarios. This investigation shows that Spain's acute stresses are higher with increasing COVID-19 damage in patients. In areas with a greater impact from the pandemic, the incidence of COVID-19 and mortality from this disease was, as was expected, increased by acute stress (Heitzman, 2020; Relojo, 2011).

Those who operate in the areas where the pandemic has been particularly active display more extreme emotional reactions because of the circumstances they are experiencing in those elements relating with thoughts, worries and bodily reactions. These emotional responses could not be established at later levels because they were emotionally obstructed to think and decide or because they were hard to understand the suffering of patients. Distress thus seemed to be linked to pandemic stresses and demands, but it was not clear whether an increase in resource availability or support services may have reduced their impacts (Caleb \& Relojo-Howell, 2019; Ogwuche et al., 2020; Stogner et al., 2020). The developments in professionals' stress responses are mostly consistent with the phases outlined by the model psychological catastrophe response (Bautista et al., 2018). The level of acute stress in the disappointment phase displayed by professionals is higher than that during the impact period. This is confirmed by the expected results and suggests that the ability to cope with a fresh outbreak will be reduced if there is not enough time between the outbreaks to allow for recovery.

\section{Wuhan Study on COVID-19 and Immediate Health Worker's Psychological Impact}

The onset of COVID-19 has put healthcare personnel under extraordinary mental stress. The objective of this study is to examine the immediate psychological effects of Tongji Hospital in Wuhan, China on the healthcare personnel. In the most countless and focused cases of infection, 
Wuhan is the epicentre of COVID-19 outbreak. With the unprecedented Wuhan lockdown, the increasing number of illnesses and deaths can produce, spread public fear and panic (Buchtele et Vol. 15, No. 1, 2021 al., 2020). Health care personnel at the Tongii Hospital may be suffering considerable psychological difficulties. They are officially classified as 'the specific hospital for the treatment of severe patients with COVID-19 in Wuhan'.

Between 8th-10th February 2020, a single centre, cross-sectional health care professionals survey was carried out utilising online questionnaires. It assessed the effect of Event Scale-Revised (IES-R), Patient Health Questionnaire-9 (PHQ-9) and Generalized 7-item (GAD-7) anxiety disorder correspondingly stress, depression, and anxiety. A survey is also meant to evaluate the effects of the Tongii Hospital's psychological safeguards. In order to find predictors of acute stress, depression and anxiety, multivariate logistic regression is utilised. A total of 5,062 surveys filled (response rate, $77.1 \%$ ) were received. The health workers reported symptoms of stress, despair, and anxiety $1509(29.8 \%), 681(13.5 \%)$ and 1218 (24.1\%) (Chen et al., 2020). Women (hazard ratio $[\mathrm{HR}], 1.32 ; p=0.032)$, working years $>10$ years $(\mathrm{HR}, 2.02 ; p<.001)$, concurrent chronic illnesses (HR, $1.51 ; p<.001$ ), history of mental illness (HR, 3.27; $p<.001$ ), and family members or family members or relatives confirmed or suspicious (HR), respectively (HR, $1.23 ; p=0.030)$. Hospital and departmental care (odds ratio [OR], 0.76; $p=0.024$ ) and full co-corporation were risk factors for stress (Relojo, 2017). A 2003 research of 1,257 health workers during the SARS outbreak of Taiwan reported anxiety and worry, with $77.4 \%$ expressed depression, and $74.2 \%$ expressed depression. In addition, a study including 652 medical leaders has shown that $68 \%$ of participants had significant levels of job-related stress, and 57.0\% had psychological discomfort during the 2003 outbreak of the Hong Kong SARS. The morbidity and anxiety of HWs in this study, as compared with the results of previous studies in hospitals affected by SARS, was relatively lower in this survey. This could be associated with the various measurements used in these surveys and the psychological protection measures implemented in the early stage by the COVID-19 management of Tongji Hospital.

The social demographic features included a woman who has history of mental health disorders, record of non-communicable physiological chronic diseases, household members or relatives reported, or confirmed COVID-19, and years of work $>10$ years. Gender variables and concurrent chronic noncommunicable diseases were examined and numerous research studies showed a greater risk of depression, anxiety and psychological stress for females and people suffering from concurrent chronic, non-communicable conditions (Nunes et al., 2016). In addition, our study demonstrated that with growing years of work, anxiety, depression, and acute stress have tended to increase, probably as most HWs were single ( $70 \%$ of health workers with working years $<2$ years are single) and have less occupational weariness and family responsibilities. It was revealed that $21.0 \%$ of sick people during the SARS were health professionals, whereas Wuhan contains about $4 \%$ of infected health workers. The present study identified or allegedly suspected $158(3.1 \%)$ health workers, 70 of whom $(44.3 \%)$ had families and family members affected. In addition, 693 (15.1\%) medical employees have not been affected, but COVID-19 has been diagnosed or suspected of their family and relations. The risk of depression was considerably higher after infected by the COVID-19 virus and raised anxiety, depression, and stress risks after the COVID-19 virus infected family members or relatives. This study shows that nurses and healthcare professionals had greater rates of psychological stress than doctors, perhaps because they have more and more close contact with patients, in line with the results of earlier studies in influenza-affected and H1N1 SARS-affected institutions. Meanwhile, it is determined that health care professionals in insulated wards have a greater risk of stress, in keeping with the results of prior studies in the SARS isolation unit. The risk of stress was higher among those with two or more children, perhaps as a result of their increased household duties. The bulk of health professionals are female, since much research have proved the problem of working women.

\section{Study on Mental Health for Physicians in the Middle of a Pandemic of COVID 19}

This research sought to evaluate the fatigue, rehabilitation, depression, anxiety and stress of physicians and to explore their relationship with desire for work and recovery experiences. This was a 
OF PSYCHOLOGY

IN THE $21^{\text {st }}$ CENTURY Vol. 15, No. 1, 2021

22

cross-sectional research among all doctors in all health facilities of the government in Selangor, Malaysia (Idris et al., 2021). Data acquired by employing self-reported questions on an internet medium in May 2020 right after the COVID-19 contagion peak in Malaysia. There were 1,050 physicians in total. The bulk of participants were non-resident non-specialist physicians (55.7\%). Mental demand $(M=7.54 ; S D=1.998)$ was the largest demand in the work environment, while detachments were the lowest demand for recuperation $(M=9.22 ; S D=5.043)$. A higher level of acute tiredness $(M=$ 63.33; $S D=19.025)$ was observed than chronic $(M=49.37, S D+24.473)$ and intershift recovery $(M=49.97, S D=19.480)$. Most of them experienced no despair, no anxiousness, no tension, and no depression (69\%). In general, higher demands on employment and less recuperation experiences were linked to poorer mental health. Emotional demands were, for example, positively linked to acute tiredness.

The objective of this study was to evaluate the fatigue, recovery, sadness, anxiety and stress levels of physicians and to determine the impact of work requests and recovery experiences with the degree of mental health of physicians. Participants were often considerably high in acute tiredness compared with chronic fatigue, adaptable recovery and typical levels of depression, anxiety and stress. This study also revealed that the need for mental employment during the pandemic was the highest, followed by time demand, emotional demand and physical demand. As for recuperation, individuals reported the strongest control over leisure time, but the lowest level of psychological separation from work events. In the middle of the COVID-19 pandemic, demands for work and regeneration are important responsibilities for the mental health of physicians. However, psychological detachment's recovery experience from work shows an important relation with the criteria of mental health in the unexpected opposite direction. In general, our results on the prevalence of depressions, anxiety and stress in doctors in other nations were similar to the general population who had lock-down procedures. This could physiologically be owing to changes in sunshine exposures during sunset, which lead to a decrease in serotonin levels related with emotional problems, such as anxiety and depression (Dubourdieu \& Escudero, 2021). Psychologically, it may be caused by a sudden disturbance of life rhythms, frequent internet connection, avoidance of pair pressure activity, or economic troubles. However, the findings revealed in our study could be underestimated since clinicians may refuse to admit the psychological problems stated in the surveys. However, an important number of doctors reported mild or severe depression, anxiety and stress in this study. Working in the middle of the COVID 19 epidemic, these are expected to take long hours, to maintain the obligation as social and moral physicians and to face a high danger of self-infection or of causing others to become infected (Boltivets \& Relojo, 2019). There is little quantitative fatigue studies and their recovery, especially among doctors or other health workers. The results of this study are, however, comparable with the results of other investigations by health workers worldwide. The heavy workload, the protracted usage of protective devices and limited recovery opportunities can attribute qualitative tiredness. A study by Sasangohar et al. (2020) showed four probable fatigue reasons, i.e., occupational hazards, enormous scaling, process failures and budgetary constraints. In the middle of the COVID-19 pandemic more fatigue-related studies are needed to determine the history, processes and results of exhaustion, given the relatively larger weight of exhaustion in relation to depression, anxiety and stress among health care workers and doctors in our study in particular. Moreover, these findings require an early and targeted intervention between high-risk front liner groups (Relojo, 2018), especially medical physicians, to avert repercussions of exhaustion. Although many people can understand tired as signals of illness, the stakeholders should see it as a sign of resource management failure.

\section{Conclusions}

Increased risk of acute exhaustion, chronic tiredness, a poor inter-shift recovery, depression, anxiety and stress were discovered, for instance, to individuals with greater emotional demand. Based on the notion of resource conservation, work demands employ personal resources such as energy to demonstrate their decline in mental health as a consequence. This result is compatible with the empiric finding that has been positively associated to stress between nurses, who have found that long working time, which is in line with high demands for work. It should be highlighted, though, that the cross-sectional design of this current study could not deduce the cause and it is therefore unknown 
who comes first, whether demand or mental health is required. Despite the highest demands of participants for work, we observed that mental demand was not significantly associated with mental health Vol. 15, No. 1, 2021 characteristics. It should be noted that this does not signify statistically that there is evidence of no partnership between them but rather that there is no data to support the link. It should be noted. This could be because of unchecked confounders, such as a single cognitive assessment of mental load. Mental demand may be seen in this context as an obstacle that involves excessive limitations and has a bad effect on mental health or is considered a challenge to personal development and reward (Kuha et al., 2019). In terms of recovery experiences, a higher control, relaxation and control level were strongly linked to a decreased risk of numerous parameters of mental health which is the desired direction. According to the notion of conserving resources, resource-intensive persons, including a greater level of recovery experiences, tend to retain or recover exhausted resources driven by demand. However, our research indicated that the psychological distinctions from the task are linked to mental health in the opposite direction. This may be because of the lockdown's contradictory effect. The mobility control order was among the severe measures to flatten the COVID-19 infection curve. During this period health employees, especially physicians, were seen to be important occupations that were not substantially susceptible to the lockdown principle. During this crisis, therefore, working seems to be more 'friendly' and less burdensome than it appears to be 'contained' at home because of its flexibility. This is perhaps the reason why work detachment is in a positive direction with results in mental health, although that was not proved empirically. The cross-sectional design of this study cannot infer causation, like the preceding talks. People with poor mental health can be assumed to be separate from their work, irrespective of their time and place. The 'principle of despair' can be explained by the notion of resource conservation in which people who have depleted personal capacity quit working to rebuild their reduced resources.

\section{References}

Acharya, S., \& Relojo, D. (2017). Examining the role of cognitive distortion and parental bonding in depressive symptoms among male adolescents: A randomised crossover trial. Journal of Innovation in Psychology, Education and Didactics, 21(1), 7-20. https://doi.org/d9m6

Antoniou, A. S. G., Davidson, M. J., \& Cooper, C. L. (2003). Occupational stress, job satisfaction and health state in male and female junior hospital doctors in Greece. Journal of Managerial Psychology, 18(6), 592-621. https://doi.org/10.1108/02683940310494403

Bautista, L., Relojo, D., Pilao, S.J., Tubon, G., \& Andal, M. (2018). Link between lifestyle and self-regulated development as components of academic performance: Basis for a psychoeducational intervention. Journal on Educational Sciences \& Psychology, 8(52), 68-72. https://doi.org/f8nb

Boltivets, S., \& Relojo, D. (2019). Psychophysiology of communication and its impact on human information processing and self-regulation: A case study in Ukraine. Archives of Psychiatry and Behavioral Sciences, 2(1), 13-21. https://doi.org/f834

Brooks, S. K., Webster, R. K., Smith, L. E., Woodland, L., Wessely, S., Greenberg, N., \& Rubin, G. J. (2020). The psychological impact of quarantine and how to reduce it: Rapid review of the evidence. The Lancet, 395(10227), 912-920. https://doi.org/10.1016/s0140-6736(20)30460-8

Buchtele, N., Rabitsch, W., Knaus, H. A., \& Wohlfarth, P. (2020). Containment of a traceable COVID-19 outbreak among healthcare workers at a hematopoietic stem cell transplantation unit. Bone Marrow Transplantation, 55(7), 1491-1492. https://doi.org/10.1038/s41409-020-0958-6

Caleb, O., \& Relojo-Howell, D. (2019). Perceived organisational support and prosocial behaviour on workplace commitment among Nigerian bank employees. CPQ Neurology and Psychology, 1(4), 1-6. https://doi.org/f8nf

Chavez-Valdez, Sarah Margarita; Esparza del Villar, Oscar Armando; Velasco Moreno, Leticia Rio; \& RelojoHowell, D. (2019). Eight crime factor model for youths in Mexican violence context. Psychology \& Society, 2(2019), 98-107. https://doi.org/10.35774/pis2019.02.098

Chekole, Y. A., Yimer Minaye, S., Mekonnen Abate, S., \& Mekuriaw, B. (2020). Perceived Stress and Its Associated 
OF PSYCHOLOGY

IN THE $21^{\text {st }}$ CENTURY Vol. 15, No. 1, 2021
Factors during COVID-19 among healthcare providers in Ethiopia: A cross-sectional study. Advances in Public Health, 2020, 1-7. https://doi.org/10.1155/2020/5036861

Chen, R. N., Liang, S. W., Peng, Y., Li, X. G., Chen, J. B., Tang, S. Y., \& Zhao, J. B. (2020). Mental health status and change in living rhythms among college students in China during the COVID-19 pandemic: A large-scale survey. Journal of Psychosomatic Research, 137, 110219. https://doi.org/10.1016/j.jpsychores.2020.110219

Chowdhury, S. R., Sunna, T. C., \& Ahmed, S. (2021). Telemedicine is an important aspect of healthcare services amid COVID-19 outbreak: Its barriers in Bangladesh and strategies to overcome. The International Journal of Health Planning and Management, 36(1), 4-12. https://doi.org/10.1002/hpm.3064

Coffey, M. (1999). Stress and burnout in forensic community mental health nurses: An investigation of its causes and effects. Journal of Psychiatric and Mental Health Nursing, 6(6), 433-443. https://doi.org/10.1046/j.1365-2850.1999.00243.x

Cohen, J. S., \& Patten, S. (2005). Well-being in residency training: a survey examining resident physician satisfaction both within and outside of residency training and mental health in Alberta. BMC Medical Education, 5(1), 1-11. https://doi.org/10.1186/1472-6920-5-21

Dubourdieu, M., \& Escudero, C. (2021). Caring for those who care Biopsychoeducation for health care personnel from psychoneuroimmunoendocrinology (PNIE) and integrative psychotherapy PNIE (PI PNIE). EC Nursing and Healthcare, 3, 150-167.

Fu, W., Wang, C., Zou, L., Guo, Y., Lu, Z., Yan, S., \& Mao, J. (2020). Psychological health, sleep quality, and coping styles to stress facing the COVID-19 in Wuhan, China. Translational Psychiatry, 10(1), 1-9. https://doi.org/10.1038/s41398-020-00913-3

Galea, S., \& Keyes, K. (2020). Understanding the Covid-19 pandemic through the lens of population health science. American Journal of Epidemiology, 189(11), 1232-1237. https://doi.org/10.1093/aje/kwaa142

Heitzman, J. (2020). Impact of COVID-19 pandemic on mental health. Psychiatria Polska, 54(2), 187-198. https://doi.org/10.12740/pp/120373

Hui, D. S., Lee, N., \& Chan, P. K. (2010). Clinical management of pandemic 2009 influenza A (H1N1) infection. Chest, 137(4), 916-925. https://doi.org/10.1378/chest.09-2344

Idris, S. H., Raduan, N. J. N., Hashim, N. A., Jauhari, N. T., \& Subbian, R. M. (2021). The intersection of COVID-19 and mental health: What's the matter with ethics? Yuridika, 36(2), 477-492. https://doi.org/10.20473/ydk.v36i2.27132

Khandelwal, S. K. (2020). Debating the process, impact, and handling of social and health determinants of the COVID-19 pandemic. Indian Journal of Social Psychiatry, 36(5), 64. https://doi.org/10.4103/ijsp.ijsp_226_20

Kuha, A., Keawkubthong, H., \& Relojo, D. (2018). The development of professional competency of teachers in Thailand: Meanings and implications. Psychreg Journal of Psychology, 2(2), 96-104. https://doi.org/f8nj

Laposa, J. M., Alden, L. E., \& Fullerton, L. M. (2003). Work stress and posttraumatic stress disorder in ED nurses/ personnel (CE). Journal of Emergency Nursing, 29(1), 23-28. https://doi.org/10.1067/men.2003.7

Marques, G., Drissi, N., de la Torre Díez, I., de Abajo, B. S., \& Ouhbi, S. (2021). Impact of COVID-19 on the psychological health of university students in Spain and their attitudes toward Mobile mental health solutions. International Journal of Medical Informatics, 147, 104369. https://doi.org/10.1016/j.ijmedinf.2020.104369

Nunes, H. E. G., Gonçalves, E. C. D. A., Vieira, J. A. J., \& Silva, D. A. S. (2016). Clustering of risk factors for non-communicable diseases among adolescents from southern Brazil. PLOS One, 11(7), e0159037. https://doi.org/10.1371/journal.pone.0159037

Ogwuche, C.H., Caleb, O., \& Relojo-Howell, D. (2020). Perceived stress and social support as predictors of subjective well-being among university students in Nigeria. Psychology \& Society, 1(79), 120-125. https://doi.org/10.35774/pis2020.01.120

Pilao, S.J., Dela Cruz, M.D., \& Relojo-Howell, D. (2019). The organisational learning of manpower service cooperative as basis for organisational development plan. CPQ Neurology and Psychology, 1(5), 1-7.

Pilao, S.J., Villanueva, A., Gornez, G.R., Villanueva, J.M., \& Relojo, D. (2017). Exploring wellness and quality of life among the elderly as a basis for a nursing care plan and psychosocial intervention. $i$-manager's Journal on Nursing, 7(3), 8-15. https://doi.org/f83x 
Pinto-Coelho, A. \& Relojo, D. (2017). Overview of utilisation of mental health services in Portugal. Journal of Innovation in Psychology, Education and Didactics, 21(1), 57-68. https://doi.org/fkht

Relojo, D. (2011). Adversity quotient and spiritual quotient and their impact to teaching performance evaluations. CEU School of Science and Technology Research Journal, 9, 62-69.

Relojo, D. (2017). Interview with Bruce Cohen, Author of 'Psychiatric Hegemony: A Marxist Theory of Mental Illness'. Psychreg Journal of Psychology, 1(1), 80-82. https://doi.org/ghhq

Relojo, D. (2018). The role of blog psychology in online mental health movement: Current status and implications. Problems of Psychology in the 21st Century, 12(1), 4-7. https://doi.org/10.33225/10.33225/ppc/18.12.04

Relojo-Howell, D. \& Stoyanova, S. (2019). Expressive writing as an anxiety-reduction intervention on test anxiety and the mediating role of first language and self-criticism in a Bulgarian sample. Journal of Educational Sciences \& Psychology, 1(4), 1-6. https://doi.org/ghhp

Relojo-Howell, D. (2020). Book review of 'Wales High School: First Diagnosis'. Psychreg Journal of Psychology, 4(2), 140-142. https://doi.org/fjcc

Sanghera, J., Pattani, N., Hashmi, Y., Varley, K. F., Cheruvu, M. S., Bradley, A., \& Burke, J. R. (2020). The impact of SARS-CoV-2 on the mental health of healthcare workers in a hospital setting - A systematic review. Journal of Occupational Health, 62(1), e12175. https://doi.org/10.1002/1348-9585.12175

Sasangohar, F., Jones, S. L., Masud, F. N., Vahidy, F. S., \& Kash, B. A. (2020). Provider burnout and fatigue during the COVID-19 pandemic: lessons learned from a high-volume intensive care unit. Anesthesia and Analgesia, 131(1), 106-111. https://doi.org/10.1213/ane.0000000000004866

Stogner, J., Miller, B. L., \& McLean, K. (2020). Police stress, mental health, and resiliency during the COVID-19 pandemic. AmericanJournal of Criminal Justice, 45(4), 718-730. https://doi.org/10.1007/s12103-020-09548-y

Vetter, M. L., Herring, S. J., Sood, M., Shah, N. R., \& Kalet, A. L. (2008). What do resident physicians know about nutrition? An evaluation of attitudes, self-perceived proficiency and knowledge. Journal of the American College of Nutrition, 27(2), 287-298. https://doi.org/10.1080/07315724.2008.10719702

Dennis Relojo-Howell (Corresponding author)
MSc, Founder, Psychreg, 2nd Floor College House, 17 King Edwards

Road, Ruislip, London, United Kingdom.

E-mail: dennis@psychreg.org

ORCID: https://orcid.org/0000-0001-8898-2077 